\title{
FUGLEDE'S THEOREM AND LIMITS OF SPECTRAL OPERATORS
}

\author{
DONALD W. HADWIN
}

\begin{abstract}
Suppose $K$ is a compact subset of the plane. A bounded sequence $\left\{\tau_{n}\right\}$ of unital homomorphisms from $C(K)$ into a Banach algebra is pointwise norm convergent if and only if $\left\{\tau_{n}(\theta(z)=z)\right\}$ is convergent. Applications are made to norm limits of scalar type spectral operators. The proof is based on an asymptotic version of Fuglede's theorem for Banach algebras.
\end{abstract}

1. Introduction. If $\left\{T_{n}\right\}$ is a sequence of normal operators on a Hilbert space and if $\left\|T_{n}-T\right\| \rightarrow 0$, then it is easy to show that $\left\|\varphi\left(T_{n}\right)-\varphi(T)\right\| \rightarrow 0$ for every continuous complex function $\varphi$. (First consider the case when $\varphi(z)$ is a polynomial in $z$ and $\bar{z}$, and then use the Stone-Weierstrass theorem.) This idea can be reformulated in terms of *-homomorphisms. Let $C(K)$ denote the $C^{*}$-algebra of all continuous complex functions on a compact subset $K$ of the plane, and let $\theta$ be the function on $K$ defined by $\theta(z)=z$ for every $z$ in $K$. Here is the reformulation: if $\left\{\tau_{n}\right\}$ is a sequence of unital *-homomorphisms from $C(K)$ into the set of operators on a Hilbert space, and if $\left\{\tau_{n}(\theta)\right\}$ is norm convergent, then there is a homomorphism $\tau$ on $C(K)$ such that $\left\|\tau_{n}(\varphi)-\tau(\varphi)\right\| \rightarrow 0$ for every $\varphi$ in $C(K)$.

It is reasonable to ask whether this phenomenon occurs for a sequence of unital homomorphisms from $C(K)$ into a Banach algebra. The uniform boundedness theorem requires that the sequence of homomorphisms be norm bounded. The main theorem of this paper (Theorem 3.1) says that if $\left\{\tau_{n}\right\}$ is a bounded sequence of unital homomorphisms from $C(K)$ into a Banach algebra, and if $\left\{\tau_{n}(\theta)\right\}$ is norm convergent, then there is a unital homomorphism $\tau$ on $C(K)$ such that $\left\|\tau_{n}(\varphi)-\tau(\varphi)\right\| \rightarrow 0$ for every $\varphi$ in $C(K)$. The proof is based upon an asymptotic Fuglede theorem for Banach algebras (Theorem 2.3).

An immediate consequence of these results is that if $\left\{T_{n}\right\}$ is a sequence of scalar type spectral operators on a weakly (sequentially) complete Banach space, if the norms of the spectral measures are uniformly bounded, and if $\left\|T_{n}-T\right\| \rightarrow 0$, then $T$ is a scalar type spectral operator and $\left\|\varphi\left(T_{n}\right)-\varphi(T)\right\|$ $\rightarrow 0$ for every continuous complex function $\varphi$.

Received by the editors April 14, 1977 and, in revised form, July 18, 1977.

AMS (MOS) subject classifications (1970). Primary 46H15, 47B40, 47A60; Secondary 47B15. Key words and phrases. Unital Banach algebra, unital homomorphism, scalar type spectral operator, Fuglede's theorem, spectral measure, Hilbert space, normal operator. 
Throughout, $K$ denotes a nonempty compact subset of the plane, $C(K)$ denotes the set of continuous complex functions on $K$, and $\theta$ denotes the function on $K$ defined by $\theta(z)=z$. Also $\mathscr{A}$ denotes a unital complex Banach algebra. If $X$ is a Banach space, then $X^{*}$ denotes its dual, $B(X)$ denotes the set of operators on $X$, and $T^{*}$ denotes the adjoint of an operator $T$ on $X$.

2. Fuglede's theorem. A well-known theorem of B. Fuglede [2] says that if $S, T$ are operators on a Hilbert space, $T$ is normal, and $S T=T S$, then $S T^{*}=T^{*} S$. The following theorem is the analogue for homomorphisms on $C(K)$. The proof is only a slight modification of M. Rosenblum's proof [6] of Fuglede's theorem. A version of C. R. Putnam's extension [5] of Fuglede's theorem is also true for homomorphisms (as is the "Putnam" analogue of Theorem 2.3). Note that Corollary 2.2 shows that a scalar type spectral operator has a unique spectral measure (see [1, Corollary XV.3.8]).

THEOREM 2.1. If $\tau$ is a continuous unital homomorphism from $C(K)$ into $\mathfrak{A}$, and if $a$ is an element of $\mathfrak{A}$ such that $a \tau(\theta)=\tau(\theta) a$, then $a \tau(\bar{\theta})=\tau(\bar{\theta}) a$.

Proof. Since $\tau(\theta)$ commutes with $a$, it follows that $\exp (i \bar{\lambda} \tau(\theta))$ commutes with $a$ for every complex number $\lambda$. Therefore

$$
\begin{aligned}
F(\lambda) & =\exp (i \lambda \tau(\bar{\theta})) a \exp (-i \lambda \tau(\bar{\theta})) \\
& =\exp [i(\bar{\lambda} \tau(\theta)+\lambda \tau(\bar{\theta}))] a \exp [-i(\bar{\lambda} \tau(\theta)+\lambda \tau(\bar{\theta}))]
\end{aligned}
$$

for every complex number $\lambda$. Since $\|\exp [ \pm i(\bar{\lambda} \tau(\theta)+\lambda \tau(\bar{\theta}))]\|=$ $\|\tau(\exp [ \pm i(\bar{\lambda} \theta+\lambda \bar{\theta})])\|_{\bullet} \leqslant\|\tau\|$ for every complex number $\lambda$, it follows from Liouville's theorem that $F(\lambda)$, being a bounded entire function, must be constant. The equation $F^{\prime \prime}(0)=0$ implies that $a \tau(\bar{\theta})=\tau(\bar{\theta}) a$.

COROLlaRY 2.2. If $\tau_{1}, \tau_{2}$ are unital continuous homomorphisms from $C(K)$ into $\mathfrak{A}$, and if $\tau_{1}(\theta)=\tau_{2}(\theta)$, then $\tau_{1}=\tau_{2}$.

Proof. Let $\mathfrak{B}$ denote the Banach algebra of $2 \times 2$ matrices with entries from $\mathfrak{A}$, equipped with any algebra norm that gives that topology of coordinatewise convergence. Define a continuous unital homomorphism $\tau$ : $C(K) \rightarrow \mathfrak{B}$ by

$$
\tau(\varphi)=\left(\begin{array}{cc}
\tau_{1}(\varphi) & 0 \\
0 & \tau_{2}(\varphi)
\end{array}\right)
$$

for every $\varphi$ in $C(K)$. Since $\tau(\theta)$ commutes with $\left(\begin{array}{l}0 \\ 0\end{array}\right)$, it follows from Theorem 2.1 that $\tau_{1}(\bar{\theta})=\tau_{2}(\bar{\theta})$. Thus, by the Stone-Weierstrass theorem, $\tau_{1}=\tau_{2}$.

The preceding theorem implies an asymptotic version of itself. The first asymptotic version of Fuglede's theorem was proved by R. Moore [4]. Note that Corollary 2.5 was proved by M. Radjabalipour and independently by $\mathrm{W}$. Zame. Let $l^{\infty}(\mathfrak{H})$ denote the Banach algebra of all bounded sequences in $\mathfrak{A}$ (with the supremum norm), and let $c_{0}(\mathfrak{H})$ denote the ideal of $l^{\infty}(\mathfrak{A})$ consisting of those sequences that converge to 0 . For $\left\{a_{n}\right\}$ in $l^{\infty}(\mathfrak{A})$ note that 


$$
\left\|\left\{a_{n}\right\}+c_{0}(\mathfrak{U})\right\|=\lim \sup \left\|a_{n}\right\| .
$$

TheOREM 2.3. Suppose $\left\{a_{n}\right\},\left\{a_{n}^{\prime}\right\}$ are bounded sequences in $\mathfrak{A}$ and $M$ is $a$ positive number such that

$$
\lim \sup \left\|p\left(a_{n}, a_{n}^{\prime}\right)\right\| \leqslant M \sup \{|p(z, \bar{z})|: z \in K\}
$$

for every noncommutative polynomial $p(x, y)$. If $\left\{b_{n}\right\}$ is a bounded sequence in $\mathfrak{A}$ such that

$$
\left\|a_{n} b_{n}-b_{n} a_{n}\right\| \rightarrow 0
$$

then

$$
\left\|a_{n}^{\prime} b_{n}-b_{n} a_{n}^{\prime}\right\| \rightarrow 0 \text {. }
$$

Proof. Statement (1) allows us to define a continuous unital homomorphism $\tau: C(K) \rightarrow l^{\infty}(\mathfrak{A}) / c_{0}(\mathfrak{A})$ that sends each polynomial $p(z, \bar{z})$ onto (the equivalence class of) the sequence $\left\{p\left(a_{n}, a_{n}^{\prime}\right)\right\}$. Statement (2) says that the sequence $\left\{b_{n}\right\}$ commutes with $\tau(\theta)$ in $l^{\infty}(\mathfrak{A}) / c_{0}(\mathfrak{A})$. Statement (3) is therefore an immediate consequence of Theorem 2.1 (i.e., $\left\{b_{n}\right\}$ commutes with $\tau(\bar{\theta})$ in $\left.l^{\infty}(\mathfrak{A}) / c_{0}(\mathfrak{U})\right)$.

COROLlaRY 2.4. If $\left\{\tau_{n}\right\}$ is a bounded sequence of unital homomorphisms from $C(K)$ into $\mathfrak{A}$, and if $\left\{b_{n}\right\}$ is a bounded sequence in $\mathfrak{A}$ such that

$$
\left\|b_{n} \tau_{n}(\theta)-\tau_{n}(\theta) b_{n}\right\| \rightarrow 0,
$$

then $\left\|b_{n} \tau_{n}(\varphi)-\tau_{n}(\varphi) b_{n}\right\| \rightarrow 0$ for every $\varphi$ in $C(K)$.

COROLlaRY 2.5. If $\left\{S_{n}\right\},\left\{T_{n}\right\}$ are bounded sequences of operators on a Hilbert space such that $\left\|T_{n}^{*} T_{n}-T_{n} T_{n}^{*}\right\| \rightarrow 0$ and $\left\|S_{n} T_{n}-T_{n} S_{n}\right\| \rightarrow 0$, then $\left\|S_{n} T_{n}^{*}-T_{n}^{*} S_{n}\right\| \rightarrow 0$.

3. The main results. We are now in a position to prove the main theorem of this paper. The proof is similar in spirit to the proof of Corollary 2.2. This theorem was proved by the author [3, Theorem 6.7] in the case when $\mathfrak{A}$ is the set of operators on a separable Hilbert space.

THEOREM 3.1. If $\left\{\tau_{n}\right\}$ is a bounded sequence of unital homomorphisms from $C(K)$ into $\mathfrak{A}$, and if $\left\{\tau_{n}(\theta)\right\}$ is norm convergent, then there is a homomorphism $\tau$ on $C(K)$ such that $\left\|\tau_{n}(\varphi)-\tau(\varphi)\right\| \rightarrow 0$ for every $\varphi$ in $C(K)$.

Proof. In view of the Stone-Weierstrass theorem we need to show only that $\left\{\tau_{n}(\bar{\theta})\right\}$ is norm convergent. Assume via contradiction that $\left\{\tau_{n}(\bar{\theta})\right\}$ is not a Cauchy sequence. By passing to a subsequence, if necessary, we can assume that there is a positive number $\varepsilon$ such that $\left\|\tau_{n+1}(\bar{\theta})-\tau_{n}(\bar{\theta})\right\| \geqslant \varepsilon$ for $n=1$, $2, \ldots$ Define a continuous homomorphism $\pi: C(K) \rightarrow B\left(l^{\infty}(\mathfrak{A})\right)$ so that, for each $\varphi$ in $C(K)$, we have that $\pi(\varphi)$ is the operator that sends a bounded sequence $\left\{a_{n}\right\}$ in $l^{\infty}(\mathfrak{A})$ onto the sequence $\left\{\tau_{n}(\varphi) a_{n}\right\}$. For each positive integer $n$, let $W_{n}$ be the operator on $l^{\infty}(\mathfrak{A})$ that interchanges the $n$th and the $(n+1)$ st terms of each sequence. Then $\left\|W_{n}\right\|=1$ for $n=1,2, \ldots$, and $\left\|W_{n} \pi(\theta)-\pi(\theta) W_{n}\right\| \rightarrow 0$. It follows from Corollary 2.4 that 


$$
\left\|\tau_{n+1}(\bar{\theta})-\tau_{n}(\bar{\theta})\right\|=\left\|W_{n} \pi(\bar{\theta})-\pi(\bar{\theta}) W_{n}\right\| \rightarrow 0 .
$$

This is the desired contradiction.

The following two corollaries follow immediately from the preceding theorem and Theorems XVII.2.4 and XVII.2.5 in [1]. These corollaries should be compared with the results in Chapter XVII of [1], especially the theorem of W. G. Bade (Theorem XVII.4.1). The reader should consult [1] for the definitions and basic facts concerning scalar type spectral operators. Note that the boundedness condition on the spectral measures is equivalent to the boundedness of the corresponding sequences of homomorphisms (see [1, p. 1929]).

COROLlARY 3.2. If $\left\{T_{n}\right\}$ is a sequence of scalar type spectral operators on a Banach space, if the spectral measures of the $T_{n}$ 's are uniformly bounded, and if $\left\|T_{n}-T\right\| \rightarrow 0$, then $T^{*}$ is a scalar type spectral operator and

$$
\left\|\varphi\left(T_{n}^{*}\right)-\varphi\left(T^{*}\right)\right\| \rightarrow 0
$$

for every continuous complex function $\varphi$.

COROLlARY 3.3. If $\left\{T_{n}\right\}$ is a sequence of scalar type spectral operators on a weakly (sequentially) complete Banach space, if the spectral measures of the $T_{n}$ 's are uniformly bounded, and if $\left\|T_{n}-T\right\| \rightarrow 0$, then $T$ is a scalar type spectral operator and $\left\|\varphi\left(T_{n}\right)-\varphi(T)\right\| \rightarrow 0$ for every continuous complex function $\varphi$.

\section{REFERENCES}

1. N. Dunford and J. T. Schwartz, Linear operators, III, Interscience, New York, 1971.

2. B. Fuglede, A commutativity theorem for normal operators, Proc. Nat. Acad. Sci. U.S.A. 36 (1950), 35-40.

3. D. W. Hadwin, An asymptotic double commutant theorem for $C^{*}$-algebras, Trans. Amer. Math. Soc. (to appear).

4. R. L. Moore, An asymptotic Fuglede theorem, Proc. Amer. Math. Soc. 50 (1975), 138-142.

5. C. R. Putnam, On normal operators in Hilbert space, Amer. J. Math. 73 (1951), 357-362.

6. M. Rosenblum, On a theorem of Fuglede and Putnam, J. London Math. Soc. 33 (1958), 376-377.

Department of Mathematics, University of New Hampshire, Durham, New Hampshire 03824 